\title{
Excited-state hadron masses using the stochastic LapH method
}

\section{John Bulava ${ }^{a}$, Justin Foley ${ }^{b}$, You-Cyuan Jhang ${ }^{c}$, Keisuke J. Juge $^{d}$, David Lenkner ${ }^{c}$,} Colin Morningstar ${ }^{* c}$, and Chik Him Wong ${ }^{e}$

${ }^{a}$ CERN, Physics Dept., 1211 Geneva 23, Switzerland

${ }^{b}$ Dept. of Physics and Astronomy, University of Utah, Salt Lake City, UT 84112, USA

${ }^{c}$ Dept. of Physics, Carnegie Mellon University, Pittsburgh, PA 15213, USA

${ }^{d}$ Dept. of Physics, University of the Pacific, Stockton, CA 95211, USA

${ }^{e}$ Dept. of Physics, University of California San Diego, La Jolla, CA 92093, USA

\begin{abstract}
Progress in computing the spectrum of excited baryons and mesons in lattice QCD is described. Large sets of spatially-extended hadron operators are used. The need for multi-hadron operators in addition to single-hadron operators is emphasized, necessitating the use of a new stochastic method of treating the low-lying modes of quark propagation which exploits Laplacian Heaviside quark-field smearing. A new glueball operator is tested and computing the mixing of this glueball operator with a quark-antiquark operator and multiple two-pion operators is shown to be feasible. Some of our initial results show warning signs about extracting high-lying resonance energies using only single-hadron operators.
\end{abstract}

The XXIX International Symposium on Lattice Field Theory - Lattice 2011

July 10-16, 2011

Squaw Valley, Lake Tahoe, California

${ }^{*}$ Speaker. 


\section{Introduction}

In a series of papers $[1,2,3,4,5,6]$, we have been striving to compute the finite-volume stationary-state energies of QCD using Markov-chain Monte Carlo integration of the QCD path integrals formulated on a space-time lattice. Such calculations are very challenging. The use of carefully designed quantum field operators is crucial for accurate determinations of low-lying energies. To study a particular state of interest, the energies of all states lying below that state must first be extracted, and as the pion gets lighter in lattice QCD simulations, more and more multi-hadron states lie below the masses of the excited resonances. The evaluation of correlations involving multi-hadron operators contains new challenges since not only must initial to final time quark propagation be included, but also final to final time quark propagation for a large number of times must be incorporated. The masses and widths of resonances must be deduced from the discrete spectrum of finite-volume stationary states for a range of box sizes.

To compute the QCD stationary-state energies, the matrices $C_{i j}(t)$ of temporal correlations of sets of single-hadron and multi-hadron operators are estimated using the Monte Carlo method. For an $N \times N$ matrix, the $N$ eigenvalues of $C\left(t_{0}\right)^{-1 / 2} C(t) C\left(t_{0}\right)^{-1 / 2}$ tend to $\exp \left(-E_{k}\left(t-t_{0}\right)\right)$ for large $t$ and fixed $t_{0}$, where the decay rates $E_{k}$ are the $N$ lowest-lying stationary-state energies that can be produced from the vacuum by the operators used. To compute the correlations involving isoscalar mesons and good multi-hadron operators, quark propagation from all spatial sites on one time slice to all spatial sites on another time slice are needed, and propagation from the sink time to the sink time are needed for a large number of sink times. A new method known as the stochastic LapH method[6] has been introduced to make such computations accurate and practical in large volumes.

\section{The Stochastic LapH method}

Finding better ways to stochastically estimate slice-to-slice quark propagators is crucial to the success of our excited-state hadron spectrum project at lighter pion masses. We have developed and tested a new scheme which combines a new way of smearing the quark field with a new way of introducing noise and dilution projectors. The new quark-field smearing scheme, here called Laplacian Heaviside (LapH), has been described in Ref. [7] and is defined by

$$
\widetilde{\psi}(x)=\Theta\left(\sigma_{s}^{2}+\widetilde{\Delta}\right) \psi(x)
$$

where $\widetilde{\Delta}$ is the three-dimensional covariant Laplacian in terms of the stout-smeared gauge field and $\sigma_{s}$ is the smearing parameter. The gauge-covariant Laplacian operator is ideal for smearing the quark field since it is one of the simplest operators that locally averages the field in such a way that all relevant symmetry transformation properties of the original field are preserved. Let $V_{\Delta}$ denote the unitary matrix whose columns are the eigenvectors of $\widetilde{\Delta}$, and let $\Lambda_{\Delta}$ denote a diagonal matrix whose elements are the eigenvalues of $\widetilde{\Delta}$ such that $\widetilde{\Delta}=V_{\Delta} \Lambda_{\Delta} V_{\Delta}^{\dagger}$. The LapH smearing matrix is then given by $\mathscr{S}=V_{\Delta} \Theta\left(\sigma_{s}^{2}+\Lambda_{\Delta}\right) V_{\Delta}^{\dagger}$. Let $V_{s}$ denote the matrix whose columns are in one-to-one correspondence with the eigenvectors associated with the $N_{v}$ lowest-lying eigenvalues of $-\widetilde{\Delta}$ on each time slice. Then our LapH smearing matrix is well approximated by the Hermitian matrix $\mathscr{S}=V_{s} V_{s}^{\dagger}$. Evaluating the temporal correlations of our hadron operators requires combining Dirac matrix elements associated with various quark lines $\mathscr{Q}$. Since we construct our hadron operators 
out of covariantly-displaced, smeared quark fields, each and every quark line involves the following product of matrices:

$$
\mathscr{Q}=D^{(j)} \mathscr{S} M^{-1} \mathscr{S} D^{(k) \dagger}
$$

where $D^{(i)}$ is a gauge-covariant displacement of type $i$.

An exact treatment of such a quark line is very costly and wasteful. Given our use of the Monte Carlo method to evaluate the path integrals over the gauge link variables, the statistical errors in our estimates of the hadron correlators are ultimately limited by the statistical fluctuations arising from the gauge-field sampling. Thus, we only need to estimate the quark lines to an accuracy comparable to the gauge noise from the Monte Carlo method. Such estimates can be obtained with far fewer inversions than required by an exact treatment of the quark lines.

Random noise vectors $\eta$ whose expectations satisfy $E\left(\eta_{i}\right)=0$ and $E\left(\eta_{i} \eta_{j}^{*}\right)=\delta_{i j}$ are useful for stochastically estimating the inverse of a large matrix $M$ as follows. Assume that for each of $N_{R}$ noise vectors, we can solve the following linear system of equations: $M X^{(r)}=\eta^{(r)}$ for $X^{(r)}$. Then $X^{(r)}=M^{-1} \eta^{(r)}$, and $E\left(X_{i} \eta_{j}^{*}\right)=M_{i j}^{-1}$ so that a Monte Carlo estimate of $M_{i j}^{-1}$ is given by $M_{i j}^{-1} \approx$ $\lim _{N_{R} \rightarrow \infty} \frac{1}{N_{R}} \sum_{r=1}^{N_{R}} X_{i}^{(r)} \eta_{j}^{(r) *}$. Unfortunately, this equation usually produces stochastic estimates with variances which are much too large to be useful. Variance reduction is done by diluting the noise vectors. A given dilution scheme can be viewed as the application of a complete set of projection operators $P^{(a)}$. Define $\eta_{k}^{[a]}=P_{k k^{\prime}}^{(a)} \eta_{k^{\prime}}$, and further define $X^{[a]}$ as the solution of $M_{i k} X_{k}^{[a]}=\eta_{i}^{[a]}$, then we have

$$
M_{i j}^{-1} \approx \lim _{N_{R} \rightarrow \infty} \frac{1}{N_{R}} \sum_{r=1}^{N_{R}} \sum_{a} X_{i}^{(r)[a]} \eta_{j}^{(r)[a] *}
$$

The use of $Z_{4}$ noise ensures zero variance in the diagonal elements $E\left(\eta_{i} \eta_{i}^{*}\right)$.

The effectiveness of the variance reduction depends on the projectors chosen. With LapH smearing, noise vectors $\rho$ can be introduced only in the LapH subspace. The noise vectors $\rho$ now have spin, time, and Laplacian eigenmode number as their indices. Color and space indices get replaced by Laplacian eigenmode number. Again, each component of $\rho$ is a random $Z_{4}$ variable so that $E(\rho)=0$ and $E\left(\rho \rho^{\dagger}\right)=I_{d}$. Dilution projectors $P^{(b)}$ are now matrices in the LapH subspace. In the stochastic LapH method, a quark line on a gauge configuration is evaluated as follows:

$$
\begin{aligned}
\mathscr{Q} & =D^{(j)} \mathscr{S} M^{-1} \mathscr{S} D^{(k) \dagger}, \\
& =D^{(j)} \mathscr{S} M^{-1} V_{s} V_{s}^{\dagger} D^{(k) \dagger}, \\
& =\sum_{b} D^{(j)} \mathscr{S} M^{-1} V_{s} P^{(b)} P^{(b) \dagger} V_{s}^{\dagger} D^{(k) \dagger}, \\
& =\sum_{b} D^{(j)} \mathscr{S} M^{-1} V_{s} P^{(b)} E\left(\rho \rho^{\dagger}\right) P^{(b) \dagger} V_{s}^{\dagger} D^{(k) \dagger}, \\
& =\sum_{b} E\left(D^{(j)} \mathscr{S} M^{-1} V_{s} P^{(b)} \rho\left(D^{(k)} V_{s} P^{(b)} \rho\right)^{\dagger}\right) .
\end{aligned}
$$

For a noise vector labelled by index $r$, displaced-smeared-diluted quark source and quark sink vectors can be defined by

$$
\begin{aligned}
& \rho^{(r)[b](j)}=D^{(j)} V_{s} P^{(b)} \rho^{(r)} \\
& \varphi^{(r)[b](j)}=D^{(j)} \mathscr{S} M^{-1} V_{s} P^{(b)} \rho^{(r)}
\end{aligned}
$$


and each quark line on a given gauge configuration can be estimated using

$$
\mathscr{Q}_{u v} \approx \frac{1}{N_{R}} \sum_{r=1}^{N_{R}} \sum_{b} \varphi_{u}^{(r)[b](j)} \rho_{v}^{(r)[b](k) *}
$$

where the subscripts $u, v$ are compound indices combining space, time, color, and spin.

Our dilution projectors are products of time dilution, spin dilution, and Laph eigenvector dilution projectors. For each type (time, spin, Laph eigenvector) of dilution, we studied four different dilution schemes. Let $N$ denote the dimension of the space of the dilution type of interest. For time dilution, $N=N_{t}$ is the number of time slices on the lattice. For spin dilution, $N=4$ is the number of Dirac spin components. For Laph eigenvector dilution, $N=N_{v}$ is the number of eigenvectors retained. The four schemes we studied are defined below:

$$
\begin{aligned}
& P_{i j}^{(a)}=\delta_{i j}, \quad a=0, \quad \text { (no dilution) } \\
& P_{i j}^{(a)}=\delta_{i j} \delta_{a i}, \quad a=0, \ldots, N-1 \quad \text { (full dilution) } \\
& P_{i j}^{(a)}=\delta_{i j} \delta_{a,\lfloor K i / N\rfloor} a=0, \ldots, K-1 \text {, (block- } K \text { ) } \\
& P_{i j}^{(a)}=\delta_{i j} \delta_{a, i \bmod K} a=0, \ldots, K-1 \text {, (interlace- } K \text { ) }
\end{aligned}
$$

where $i, j=0, \ldots, N-1$, and we assume $N / K$ is an integer. We use a triplet (T, S, L) to specify a given dilution scheme, where "T" denotes time, "S" denotes spin, and "L" denotes Laph eigenvector dilution. The schemes are denoted by 1 for no dilution, $\mathrm{F}$ for full dilution, and $\mathrm{B} K$ and $\mathrm{I} K$ for block- $K$ and interlace- $K$, respectively. For example, full time and spin dilution with interlace8 Laph eigenvector dilution is denoted by (TF, SF, LI8). Introducing diluted noise in this way produces correlation functions with significantly reduced variances, yielding nearly an order of magnitude reduction in the statistical error over previous methods. The volume dependence of this new method was found to be very mild, allowing the method to be useful on large lattices. For all forward-time quark lines, we use dilution scheme (TF, SF, LI8), and for all same-sink-time quark lines, we use (TI16, SF, LI8). Interlacing in time makes the calculation of a large number of sink-to-sink diagrams possible with a feasibly small number of Dirac matrix inversions. The use of dilution projectors that interlace in time is perhaps the most important factor behind the success of the stochastic LapH method.

Details on how the temporal correlations of hadron operators are evaluated are given in Ref. [6]. A very useful feature of the method is the fact that the hadron correlators completely factorize into a function associated with the sink time slice $t_{F}$, and another function associated with the source time slice $t_{0}$. Summations over color, spin, and spatial sites at the source can be completely separated from the color, spin, and spatial summations at the sink. The stochastic LapH method leads to complete factorization of hadron sources and sinks in temporal correlations, which greatly simplifies the logistics of evaluating correlation matrices involving large numbers of operators. Implementing the Wick contractions of the quark lines is also straightforward. Contributions from different Wick orderings within a class of quark-line diagrams differ only by permutations of the noises at either the source or the sink.

\section{First results}

Our first results for the isovector mass spectrum on a large $24^{3} \times 128$ anisotropic lattice are shown in Fig. 1. The pion mass is about $m_{\pi} \sim 390 \mathrm{MeV}$ here. These results are not finalized 


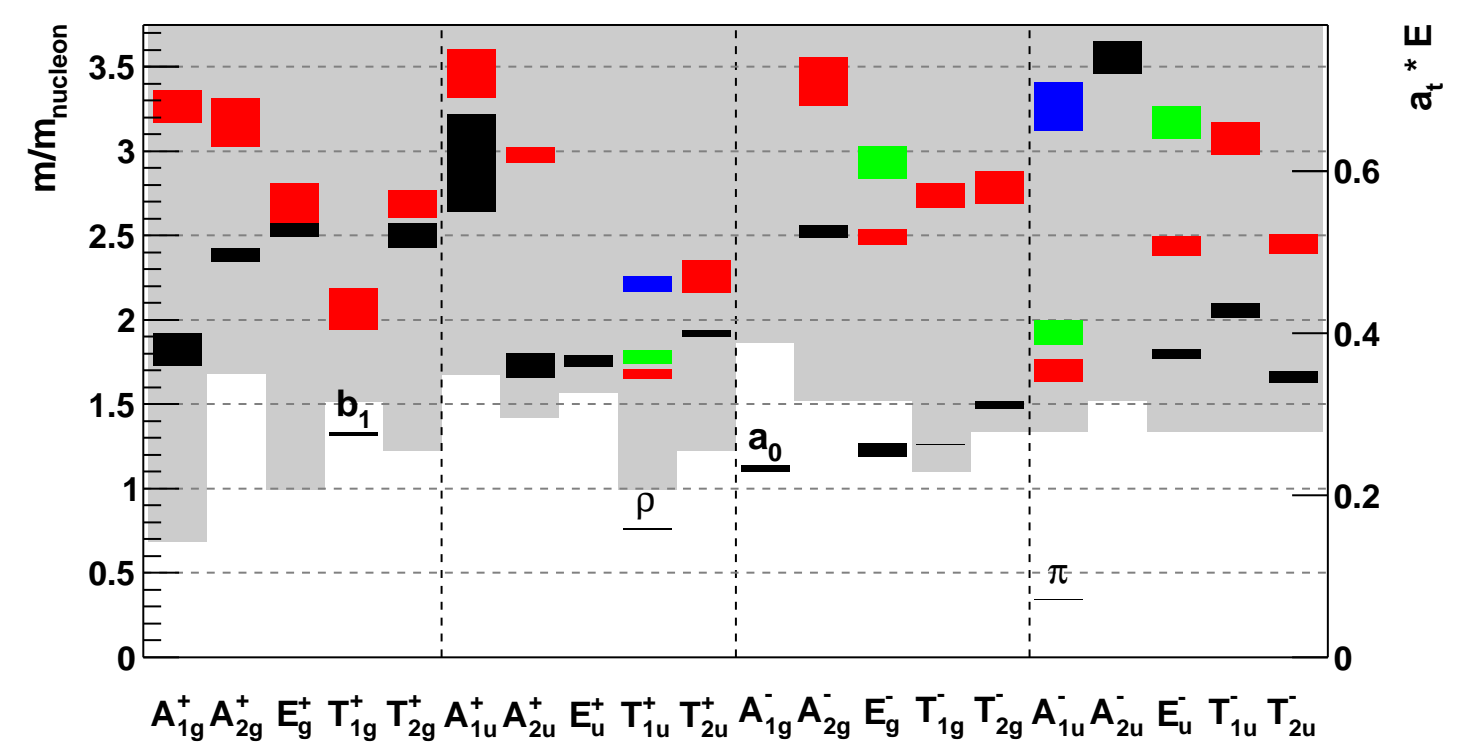

Figure 1: Masses of the isovector mesons in terms of the nucleon mass using the stochastic LapH method with 170 gauge configurations on a $24^{3} \times 128$ anisotropic lattice. The pion mass is about $m_{\pi} \sim 390 \mathrm{MeV}$. In the irrep labels, the letters with numerical subscripts refer to the point group $O_{h}$ irreps, the subscripts $g$ and $u$ refer to even and odd parity, respectively, and the superscripts \pm refer to $G$-parity. Only single-hadron operators were used with dilution scheme $(T F, S F, L I 8)$. The shaded region indicates the threshold locations for multi-hadron energy levels. We emphasize that extractions of energies in the shaded regions could be complicated by "false plateaux" unless multi-hadron operators are used.

since only single-hadron operators were used. The threshold locations for multi-hadron energy levels, assuming the interaction energies are small, are indicated by the shaded region. Extractions of energies in the shaded regions can only be considered reliable if multi-hadron operators are used, in addition to the single-hadron operators. The inclusion of the multi-hadron operators is in progress.

Some initial results that incorporate multi-hadron operators are shown in Fig. 2 . A $2 \times 2$ correlation matrix was evaluated involving a single-site $\rho$-meson operator and a total isospin $I=1 \pi \pi$ operator in a $P$-wave with minimal relative momentum. The stochastic LapH method enables very accurate estimates of all elements (both diagonal and off-diagonal) of this correlation matrix such that diagonalization can be done. The effective masses associated with the diagonalized correlator are shown in Fig. 2. The $\rho$ operator dominates the lowest-lying level, while the $\pi \pi$ operator dominates the first-excited state. Although the mixing of the operators is small, it is not negligible. This is certainly a warning about the dangers of extracting high-lying resonance energies using only single-hadron operators.

Determining meson masses in the interesting scalar isoscalar sector will ultimately involve including a scalar glueball operator, so we began looking into the feasibility of such calculations. LapH quark-field smearing involves the covariant spatial Laplacian $\widetilde{\Delta}$. The eigenvalues of the Laplacian are invariant under rotations and gauge transformations so are appropriate for a scalar glueball operator. The lowest-lying eigenvalue was studied, as well as other functions of the eigenvalues. We found that any combination of the low-lying eigenvalues worked equally well for 

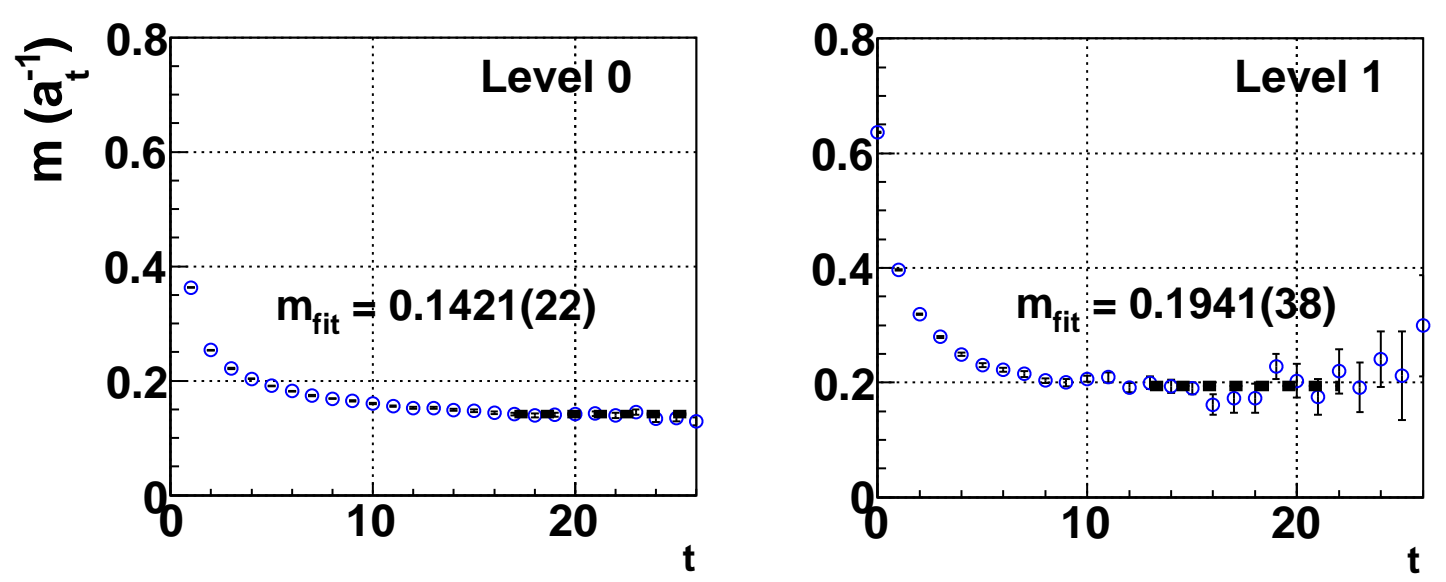

Figure 2: Effective masses corresponding to the diagonal elements of the rotated $2 \times 2$ correlator matrix involving a single-site $\rho$-meson operator and an $I=1 \pi \pi$ operator in a $P$-wave with minimal relative momentum. The $\rho$ operator dominates the lowest-lying level (left), while the $\pi \pi$ operator dominates the first-excited state (right). Although the mixing of the operators is small, it is not negligible. These results were obtained on 584 configurations of the $24^{3} \times 128$ lattice with pion mass $m_{\pi} \sim 240 \mathrm{MeV}$.
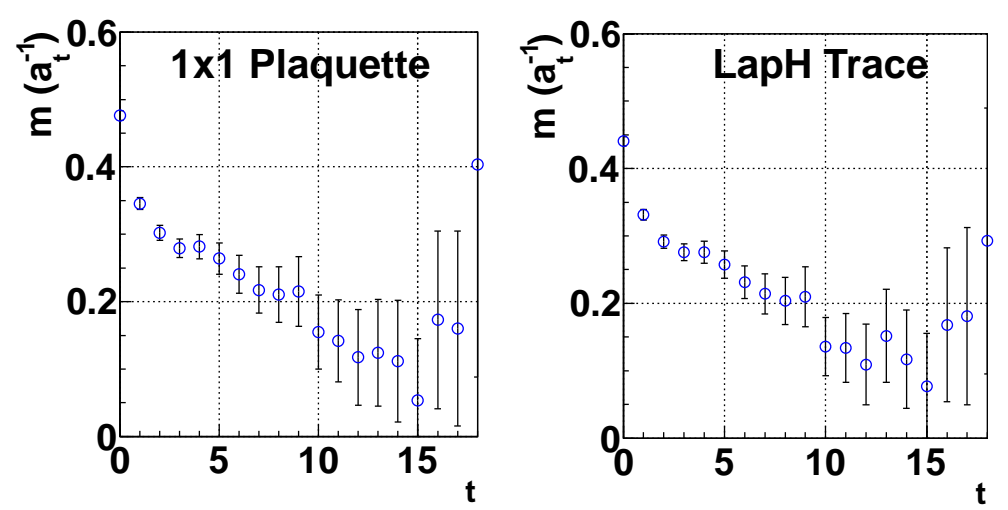

Figure 3: Comparison of the effective mass associated with the correlator of the standard smeared plaquette glueball operator (left) with that of our new glueball operator $G_{\Delta}$ defined in the text (right). The similarity of the two results shows that the new glueball operator is just as useful as the familiar smeared plaquette for studying the scalar glueball. These effective masses do not reach a plateau at the glueball mass since various $\pi \pi$ states and other multi-hadron states have smaller energies than the glueball mass. These results were obtained using 584 configs of the $24^{3}$ ensemble for pion mass $m_{\pi} \sim 240 \mathrm{MeV}$.

studying the scalar glueball. In particular, the operator defined by $G_{\Delta}(t)=-\operatorname{Tr}\left(\Theta\left(\sigma_{s}^{2}+\widetilde{\Delta}\right) \widetilde{\Delta}(t)\right)$ was used. The effective mass associated with this operator is compared to that of the smeared plaquette glueball operator in Fig. 3. The similarity of the results shows that $G_{\Delta}$ is just as useful as the familiar smeared plaquette for studying the scalar glueball.

Effective masses corresponding to the diagonalized $4 \times 4$ correlator matrix involving a scalar isoscalar single-site quark-antiquark meson operator, the new glueball operator $G_{\Delta}$, and two $I=0$ $\pi \pi$ operators in an $S$ wave (one with zero relative momentum and the other with minimal nonzero relative momentum) are shown in Fig. 4 . Mixing of these operators is sizeable. More $\pi \pi$ operators 

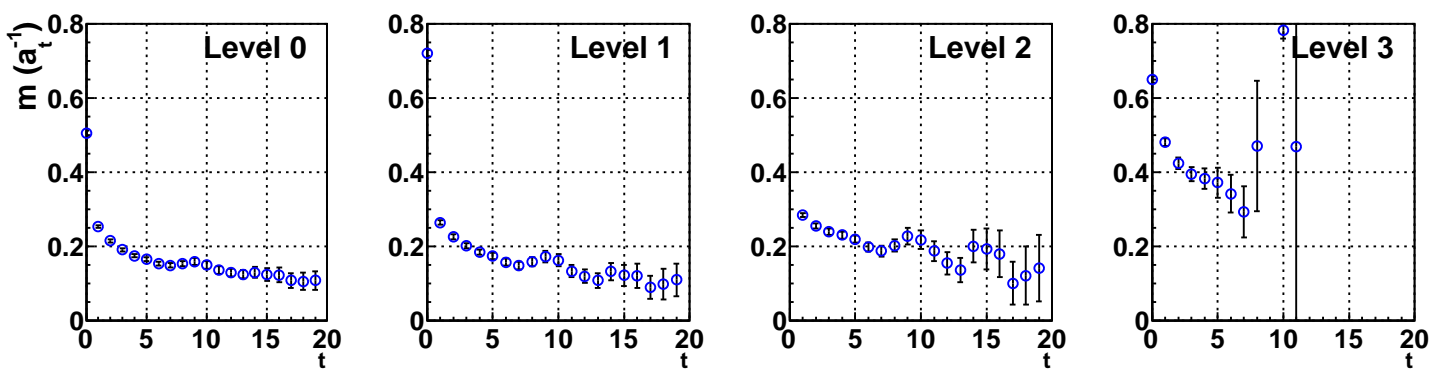

Figure 4: Effective masses corresponding to the diagonal elements of the rotated $4 \times 4$ correlator matrix involving a scalar isoscalar single-site quark-antiquark meson operator, the new glueball operator $G_{\Delta}$, and two $I=0 \pi \pi$ operators in an $S$ wave (one with zero relative momentum and the other with minimal nonzero relative momentum). Mixing of these operators is sizeable. More $\pi \pi$ operators must be included to reliably extract the glueball mass. These results were obtained on 100 configurations of a $16^{3} \times 128$ lattice with pion mass $m_{\pi} \sim 390 \mathrm{MeV}$.

must be included to reliably extract the glueball mass.

\section{Conclusion}

A new method of stochastically estimating the low-lying effects of quark propagation was proposed which allows accurate determinations of temporal correlations of single-hadron and multihadron operators in lattice QCD. The method enables accurate treatment of hadron correlators involving quark propagation from all spatial sites on one time slice to all spatial sites on another time slice. Contributions involving quark lines originating at the sink time $t_{F}$ and terminating at the same sink time $t_{F}$ are easily handled, even for a large number of $t_{F}$ times.

The effectiveness of the method can be traced to two of its key features: the use of noise dilution projectors that interlace in time and the use of $Z_{N}$ noise in the subspace defined by the Laplacian Heaviside quark-field smearing. Introducing noise in the $\mathrm{LapH}$ subspace results in greatly reduced variances in temporal correlations compared to methods that introduce noise on the entire lattice. Although the number of Laplacian eigenvectors needed to span the LapH subspace rises linearly with the spatial volume, we found that the number of inversions of the Dirac matrix needed for a target accuracy was remarkably insensitive to the lattice volume, once a sufficient number of dilution projectors were introduced.

In addition to increased efficiency, the stochastic LapH method has other advantages. The method leads to complete factorization of hadron sources and sinks in temporal correlations, which greatly simplifies the logistics of evaluating correlation matrices involving large numbers of operators. Implementing the Wick contractions of the quark lines is also straightforward. Contributions from different Wick orderings within a class of quark-line diagrams differ only by permutations of the noises at the source.

The results presented here demonstrate that the stochastic LapH method is useful for accurately estimating all of the temporal correlations needed for a full study of the QCD stationarystate energy spectrum, which we are currently pursuing. A new glueball operator was tested and computing the mixing of this glueball operator with a quark-antiquark operator and multiple two- 
pion operators was shown to be feasible. Some of our initial results showed warning signs about extracting high-lying resonance energies using only single-hadron operators.

This work was supported by the U.S. NSF under awards PHY-0510020, PHY-0653315, PHY0704171, PHY-0969863, and PHY-0970137, and through TeraGrid/XSEDE resources provided by TACC and NICS under grant numbers TG-PHY100027 and TG-MCA075017.

\section{References}

[1] S. Basak, R.G. Edwards, G.T. Fleming, U.M. Heller, C. Morningstar, D. Richards, I. Sato, S. Wallace, Phys. Rev. D 72, 094506 (2005).

[2] S. Basak, R. Edwards, G. Fleming, U.M. Heller, C. Morningstar, D. Richards, I. Sato, S. Wallace, Phys. Rev. D 72, 074501 (2005).

[3] S. Basak, R.G. Edwards, G.T. Fleming, K.J. Juge, A. Lichtl, C. Morningstar, D.G. Richards, I. Sato, S.J. Wallace, Phys. Rev. D 76, 074504 (2007).

[4] J. Bulava, R.G. Edwards, E. Engelson, J. Foley, B. Joo, A. Lichtl, H.W. Lin, N. Mathur, C. Morningstar, D.G. Richards, S. Wallace, Phys. Rev. D 79, 034505 (2009).

[5] J. Bulava, R.G. Edwards, E. Engelson, B. Joo, H-W. Lin, C. Morningstar, D.G. Richards, S.J. Wallace, Phys. Rev. D 82, 014507 (2010).

[6] C. Morningstar, J. Bulava, J. Foley, K.J. Juge, D. Lenkner, M. Peardon, C.H. Wong, Phys. Rev. D 83, 114505 (2011).

[7] M. Peardon, J. Bulava, J. Foley, C. Morningstar, J. Dudek, R.G. Edwards, B. Joo, H-W. Lin, D.G. Richards, K.J. Juge, Phys. Rev. D 80, 054506 (2009). 